\title{
Pseudo-spectral methods for the Laplace-Beltrami equation and the Hodge decomposition on surfaces of genus one*
}

\author{
Lise-Marie Imbert-Gerard $\dagger^{\dagger}$ and Leslie Greengard ${ }^{\ddagger}$
}

September 30, 2016

\begin{abstract}
The inversion of the Laplace-Beltrami operator and the computation of the Hodge decomposition of a tangential vector field on smooth surfaces arise as computational tasks in many areas of science, from computer graphics to machine learning to computational physics. Here, we present a high-order accurate pseudo-spectral approach, applicable to closed surfaces of genus one in three dimensional space, with a view toward applications in plasma physics and fluid dynamics.
\end{abstract}

\section{Introduction}

Partial differential equations on smooth manifolds [24, 31, 35] arise in many areas of science, including graphics and image analysis [6, 16, 21, 22, 30, 33, 39], machine learning [4, 8, plasma physics and fluid dynamics [3, 10, 11, 17, 25], and electromagnetic theory [7, 9, 13, 14, 15, 20, 28]. In some cases, the problem of interest concerns a physical process such as surface growth or surface diffusion [32, 34]. In others, the primary concern is with the representation of vector fields on the surface, such as currents which give rise to electromagnetic fields in the ambient space. In the latter context, it is known from Hodge theory [35, 38, that one can express any vector field $\mathbf{j}$ on a smooth closed multiply connected surface $\Gamma$ in $\mathbb{R}^{3}$ in terms of an orthogonal decomposition of the form:

$$
\mathbf{j}=\nabla_{\Gamma} \alpha+\mathbf{n} \times \nabla_{\Gamma} \beta+\mathbf{j}_{H}
$$

${ }^{*}$ This work was supported in part by the Applied Mathematical Sciences Program of the U.S. Department of Energy under contract DEFGO288ER25053 and by the Office of the Assistant Secretary of Defense for Research and Engineering and AFOSR under NSSEFF program award FA9550-10-1-0180

${ }^{\dagger}$ Courant Institute, New York University, New York, NY. Email: imbertgerard@cims.nyu.edu

${ }^{\ddagger}$ Courant Institute, New York University, and Simons Center for Data Analysis, Simons Foundation, New York, NY. Email: greengard@cims.nyu.edu 
for some scalar functions $\alpha, \beta$, where $\mathbf{n}$ is the surface normal, $\nabla_{\Gamma}$ denotes the surface gradient on $\Gamma$, and $\mathbf{j}_{H}$ is both surface divergence-free and surface curl-free, namely

$$
\operatorname{Div} \mathbf{j}_{H}=\nabla_{\Gamma} \cdot \mathbf{j}_{H}=0, \quad \operatorname{Curl} \mathbf{j}_{H}=\nabla_{\Gamma} \cdot\left(\mathbf{n} \times \mathbf{j}_{H}\right)=0 .
$$

These operators are defined formally in terms of the metric tensor in the next section. For the moment, we simply note that $C u r l \mathbf{j}$ is defined as a scalar field, corresponding to the normal component of the three dimensional curl of a suitable extension of the tangential vector field $\mathbf{j}$ in a neighborhood of the surface [9, 28]. Vector fields satisfying both equations in (2) are called harmonic vector fields and, from Hodge theory, the space of such functions is of dimension exactly $2 \mathfrak{g}$ for a surface of genus $\mathfrak{g}$ [35, 38]. We also note (for later reference) that $\operatorname{Curl}\left(\nabla_{\Gamma} \alpha\right)=0$ and $\operatorname{Div}\left(\mathbf{n} \times \nabla_{\Gamma} \beta\right)=0$.

Despite their importance, differential equations on manifolds have received much less attention from the scientific computing community than boundary value problems in Euclidean space. For some sense of the available literature, we refer the reader to [2, 12, 29] and the references therein for a discussion of finite element analysis, [1, 5, 23, 27, 32] for a discussion of finite difference methods, and [26] for a discussion of integral equation methods.

Here, we restrict our attention to smooth, closed surfaces $\Gamma$ of genus one embedded in $\mathbb{R}^{3}$, primarily because of their importance in plasma physics and stellarator design [19]. More precisely, we assume that $\Gamma$ is a smooth toroidal surface:

$$
\mathbf{x}(u, v)=(x(u, v), y(u, v), z(u, v)),
$$

where $\mathbf{x}(u, v)$ is a doubly periodic function of $(u, v) \in[0,2 \pi]^{2}$. In the case of a surface of revolution, this takes the special form

$$
\mathbf{x}(u, v)=(r(u) \cos v, r(u) \sin v, z(u)) .
$$

We will refer, in either setting, to $u$ as the poloidal angle and to $v$ as the toroidal angle.

A smooth real-valued scalar function $f(u, v)$ defined on $\Gamma$ is periodic, and it will be convenient to write $f$ in the form of a Fourier series:

$$
f(u, v)=\sum_{m=-\infty}^{\infty} \sum_{n=-\infty}^{\infty} f_{m n} e^{\imath(m u+n v)} .
$$

A smooth, real-valued tangential vector field $\mathbf{F}$ on the surface defined by (3) is generally expressed in terms of the standard, contravariant components in the standard, covariant basis:

$$
\mathbf{F}(u, v)=F^{1}(u, v) \partial_{u} \mathbf{x}(u, v)+F^{2}(u, v) \partial_{v} \mathbf{x}(u, v) .
$$

For simplicity, we will often write $\mathbf{F}=\left(F^{1}, F^{2}\right)$. Since $\mathbf{F}:\left[0,2 \pi{ }^{2} \rightarrow \mathbb{R}^{3}\right.$ is periodic, it can be represented as a Fourier series of the form

$$
\mathbf{F}(u, v)=\sum_{m=-\infty}^{\infty} \sum_{n=-\infty}^{\infty}\left(F_{m n}^{1} \partial_{u} \mathbf{x}(u, v)+F_{m n}^{2} \partial_{v} \mathbf{x}(u, v)\right) e^{\imath(m u+n v)} .
$$


The space of periodic tangential vector fields will be denoted $\mathcal{V}_{\Gamma}$ and the subspace of $\mathcal{V}_{\Gamma}$ consisting of $k$-times differentiable functions will be denoted by $\mathcal{V}_{\Gamma}^{k}$. Similarly, the space of periodic scalar functions will be denoted by $\mathcal{S}_{\Gamma}$, and its subspace of $k$-times differentiable functions by $\mathcal{S}_{\Gamma}^{k}$.

In this paper, we construct efficient, high-order pseudospectral methods for evaluating the surface divergence, gradient and curl (section 2), inverting the Laplace-Beltrami operator (section 3), determining a basis for surface harmonic vector fields (section 4), and computing the Hodge decomposition (section 5). Section 6 contains some illustrative numerical examples and section 7 contains some concluding remarks.

\section{Mathematical preliminaries}

This section is dedicated to the definition and discretization of $L^{2}$ inner products of scalar and vector-valued functions, and partial differential operators on a surface of genus 1 in $\mathbb{R}^{3}$.

\subsection{Surface operators}

For a surface $\Gamma$ defined by a parametrization (3), we will denote the metric tensor by

$$
G=\left(\begin{array}{ll}
G_{u u} & G_{u v} \\
G_{u v} & G_{v v}
\end{array}\right)
$$

where

$$
G_{u u}=\partial_{u} \mathbf{x} \cdot \partial_{u} \mathbf{x}, \quad G_{u v}=\partial_{u} \mathbf{x} \cdot \partial_{v} \mathbf{x}, \quad \text { and } \quad G_{v v}=\partial_{v} \mathbf{x} \cdot \partial_{v} \mathbf{x} .
$$

and the determinant of $G$ by $g$. By the Cauchy-Schwarz inequality, it is clear that $g \geq 0$. We assume $g>0$, so that $\left(\partial_{u} \mathbf{x}, \partial_{v} \mathbf{x}\right)$ is a basis for the tangent space to the surface at every point under the parametrization (3). The induced $L_{2}$ inner product for tangential vector fields $\mathbf{v}_{1}, \mathbf{v}_{2}$ is

$$
\left\langle\mathbf{v}_{1}, \mathbf{v}_{2}\right\rangle_{\Gamma}=\int_{\Gamma} \mathbf{v}_{1}(\mathbf{x}(u, v)) \cdot \mathbf{v}_{2}(\mathbf{x}(u, v)) d A(u, v)=\int_{0}^{2 \pi} \int_{0}^{2 \pi} \mathbf{v}_{1}(u, v) \cdot \mathbf{v}_{2}(u, v) \sqrt{g(u, v)} d u d v .
$$

The induced $L_{2}$ inner product for scalar fields $\phi, \psi$ is

$$
\langle\phi, \psi\rangle_{\Gamma}=\int_{\Gamma} \phi(\mathbf{x}(u, v)) \psi(\mathbf{x}(u, v)) d A(u, v)=\int_{0}^{2 \pi} \int_{0}^{2 \pi} \phi(u, v) \psi(u, v) \sqrt{g(u, v)} d u d v
$$

The surface normal is given by the unit vector $\mathbf{n}=\frac{1}{\sqrt{g}}\left(\partial_{u} \mathbf{x} \times \partial_{v} \mathbf{x}\right)$.

Definition 1 Assume the surface $\Gamma$ is such that $G_{u u}, G_{u v}$ and $G_{v v}$ are in $\mathcal{S}_{\Gamma}^{1}$, and that $g \neq 0$. Let $f(u, v)$ denote a scalar function and let $\mathbf{F}(u, v)$ denote a tangential vector field 
on the surface $\Gamma$. Then the surface gradient, divergence, curl and Laplace-Beltrami operator are given by [9, [28]:

$$
\begin{aligned}
\text { Grad } f & =\nabla_{\Gamma} f=\left[\frac{G_{v v}}{g} \partial_{u} f-\frac{G_{u v}}{g} \partial_{v} f\right] \partial_{u} \mathbf{x}+\left[-\frac{G_{u v}}{g} \partial_{u} f+\frac{G_{u u}}{g} \partial_{v} f\right] \partial_{v} \mathbf{x}, \forall f \in \mathcal{S}_{\Gamma}^{1}, \\
\operatorname{Div} \mathbf{F} & =\nabla_{\Gamma} \cdot \mathbf{F}=\frac{1}{\sqrt{g}}\left[\partial_{u}\left(\sqrt{g} F^{1}\right)+\partial_{v}\left(\sqrt{g} F^{2}\right)\right], \forall \mathbf{F} \in \mathcal{V}_{\Gamma}^{1}, \\
\operatorname{Curl} \mathbf{F} & =-\nabla_{\Gamma} \cdot(\mathbf{n} \times \mathbf{F})=\frac{1}{\sqrt{g}}\left(\partial_{u}\left(G_{u v} F^{1}+G_{v v} F^{2}\right)-\partial_{v}\left(G_{u u} F^{1}+G_{u v} F^{2}\right)\right), \forall \mathbf{F} \in \mathcal{V}_{\Gamma}^{1}, \\
\Delta_{\Gamma} f & =\nabla_{\Gamma} \cdot\left(\nabla_{\Gamma} f\right)=\frac{1}{\sqrt{g}}\left[\partial_{u}\left(\frac{G_{v v}}{\sqrt{g}} \partial_{u} f-\frac{G_{u v}}{\sqrt{g}} \partial_{v} f\right)+\partial_{v}\left(\frac{G_{u u}}{\sqrt{g}} \partial_{v} f-\frac{G_{u v}}{\sqrt{g}} \partial_{u} f\right)\right], \forall f \in \mathcal{S}_{\Gamma}^{2},
\end{aligned}
$$

respectively.

\subsection{Discretization}

In order to develop high-order pseudospectral methods, we assume that scalar functions, such as $f$ or $F^{1}$, are discretized on a uniform grid with $N^{2}$ points:

$$
\vec{f}=\left\{f\left(u_{k}, v_{l}\right)\right\}, \vec{F}^{1}=\left\{F^{1}\left(u_{k}, v_{l}\right)\right\},
$$

where

$$
h=\frac{2 \pi}{N}, \quad u_{k}=k h, \quad v_{l}=l h, \quad k, l=1, \ldots, N .
$$

We approximate the Fourier coefficients

$$
[\mathcal{F}(f)]_{m n} \equiv \frac{1}{(2 \pi)^{2}} \int_{0}^{2 \pi} \int_{0}^{2 \pi} f(u, v) e^{-\imath(m u+n v)} d u d v
$$

by the discrete Fourier transform $\widehat{f}=\mathcal{F}_{h}(\vec{f})$ :

$$
\widehat{f}_{m n}=\left(\frac{h}{2 \pi}\right)^{2} \sum_{k=1}^{N} \sum_{l=1}^{N} f\left(u_{k}, v_{l}\right) e^{-\imath\left(m u_{k}+n v_{l}\right)} .
$$

With a slight abuse of notation, we assume that the set of discrete values on the surface, namely $\vec{f} \in \mathbb{R}^{N^{2}}$, and the corresponding discrete Fourier transform, $\widehat{f} \in \mathbb{C}^{N^{2}}$, have been "unrolled" and can be considered column vectors of length $N^{2}$. Likewise, we will view $\left(\vec{F}^{1}, \vec{F}^{2}\right)$ or $\left(\vec{F}^{1}, \vec{F}^{2}\right)^{T}$ as column or row vectors in $\mathbb{R}^{2 N^{2}}$.

Definition 2 Given the Fourier coefficient vector $\widehat{f}$, we will denote by $\mathcal{D}_{\sigma(n, m)}$ the (Fourier space) diagonal operator such that

$$
\left[\mathcal{D}_{\sigma(n, m)} \widehat{f}\right]_{m n}=\sigma(n, m) \widehat{f}_{m n}
$$


Thus, $\left[\mathcal{D}_{i m} \widehat{f}\right]_{m n}=i m \widehat{f}_{m n}$, etc.

Given the vector $\vec{f}$, we will denote by $D_{u g}$ and $D_{v g}$ the (physical space) diagonal operators such that

$$
\left[D_{u g} \vec{f}\right]\left(u_{k}, v_{l}\right)=\frac{\partial_{u} g}{2 g}\left(u_{k}, v_{l}\right) f\left(u_{k}, v_{l}\right), \quad\left[D_{v g} \vec{f}\right]\left(u_{k}, v_{l}\right)=\frac{\partial_{v} g}{2 g}\left(u_{k}, v_{l}\right) f\left(u_{k}, v_{l}\right) .
$$
that

We will denote by $D_{u u}, D_{u u g}, D_{u v}, D_{u v g}, D_{v v}$, and $D_{v v g}$, the diagonal operators such

$$
\begin{gathered}
{\left[D_{u u} \vec{f}\right]\left(u_{k}, v_{l}\right)=\frac{G_{u u}}{g}\left(u_{k}, v_{l}\right) f\left(u_{k}, v_{l}\right), \quad\left[D_{u u g} \vec{f}\right]\left(u_{k}, v_{l}\right)=\frac{G_{u u}}{\sqrt{g}}\left(u_{k}, v_{l}\right) f\left(u_{k}, v_{l}\right),} \\
{\left[D_{u v} \vec{f}\right]\left(u_{k}, v_{l}\right)=-\frac{G_{u v}}{g}\left(u_{k}, v_{l}\right) f\left(u_{k}, v_{l}\right), \quad\left[D_{u v g} \vec{f}\right]\left(u_{k}, v_{l}\right)=\frac{G_{u v}}{\sqrt{g}}\left(u_{k}, v_{l}\right) f\left(u_{k}, v_{l}\right),} \\
{\left[D_{v v} \vec{f}\right]\left(u_{k}, v_{l}\right)=\frac{G_{v v}}{g}\left(u_{k}, v_{l}\right) f\left(u_{k}, v_{l}\right), \quad\left[D_{v v g} \vec{f}\right]\left(u_{k}, v_{l}\right)=\frac{G_{v v}}{\sqrt{g}}\left(u_{k}, v_{l}\right) f\left(u_{k}, v_{l}\right) .}
\end{gathered}
$$

We will denote by $D_{u, u v}, D_{v, u v}, D_{v, u u}$, and $D_{u, v v}$, the (physical space) diagonal operators such that

$$
\begin{aligned}
& {\left[D_{u, u v} \vec{f}\right]\left(u_{k}, v_{l}\right)=\frac{\partial_{u} G_{u v}}{\sqrt{g}}\left(u_{k}, v_{l}\right) f\left(u_{k}, v_{l}\right), \quad\left[D_{v, u v} \vec{f}\right]\left(u_{k}, v_{l}\right)=\frac{\partial_{v} G_{u v}}{\sqrt{g}}\left(u_{k}, v_{l}\right) f\left(u_{k}, v_{l}\right),} \\
& {\left[D_{v, u u} \vec{f}\right]\left(u_{k}, v_{l}\right)=\frac{\partial_{v} G_{u u}}{\sqrt{g}}\left(u_{k}, v_{l}\right) f\left(u_{k}, v_{l}\right), \quad\left[D_{u, v v} \vec{f}\right]\left(u_{k}, v_{l}\right)=\frac{\partial_{u} G_{v v}}{\sqrt{g}}\left(u_{k}, v_{l}\right) f\left(u_{k}, v_{l}\right),}
\end{aligned}
$$

Lemma 1 Let $\vec{f}$ denote a discretized scalar function on a surface $\Gamma$ and let $\overrightarrow{\mathbf{F}}=\left(\overrightarrow{F^{1}}, \overrightarrow{F^{2}}\right)^{T}$ denote a discretized tangential vector field in the standard basis. Then the surface gradient, divergence, and Laplace-Beltrami operator are approximated by:

$$
\begin{aligned}
\operatorname{Grad}_{h} \vec{f} & =\nabla_{\Gamma, h} \vec{f}=\left(\begin{array}{cc}
D_{v v} & D_{u v} \\
D_{u v} & D_{u u}
\end{array}\right)\left(\begin{array}{cc}
\mathcal{F}_{h}^{*} & 0 \\
0 & \mathcal{F}_{h}^{*}
\end{array}\right)\left(\begin{array}{l}
\mathcal{D}_{i m} \\
\mathcal{D}_{i n}
\end{array}\right) \mathcal{F}_{h} \vec{f} \\
\operatorname{Div}_{h} \overrightarrow{\mathbf{F}} & =\nabla_{\Gamma, h} \cdot \overrightarrow{\mathbf{F}}=\left(\begin{array}{ll}
V_{1}(G) & \left.V_{2}(G)\right)\left(\begin{array}{c}
\overrightarrow{F^{1}} \\
\overrightarrow{F^{2}}
\end{array}\right) \\
\operatorname{Curl}_{h} \overrightarrow{\mathbf{F}} & =\left(\begin{array}{ll}
C_{1}(G) & C_{2}(G)
\end{array}\right)\left(\begin{array}{c}
\overrightarrow{F^{1}} \\
\overrightarrow{F^{2}}
\end{array}\right) \\
\Delta_{\Gamma, h} \vec{f} & =\operatorname{Div}_{h} \operatorname{Grad}_{h} \vec{f} .
\end{array}\right.
\end{aligned}
$$


where

$$
\begin{aligned}
& V_{1}(G)=D_{u g}+\mathcal{F}_{h}^{*} \mathcal{D}_{i m} \mathcal{F}_{h} \\
& V_{2}(G)=D_{v g}+\mathcal{F}_{h}^{*} \mathcal{D}_{i n} \mathcal{F}_{h} \\
& C_{1}(G)=D_{u, u v}-D_{v, u u}+D_{u v g} \mathcal{F}_{h}^{*} \mathcal{D}_{i m} \mathcal{F}_{h}-D_{u u g} \mathcal{F}_{h}^{*} \mathcal{D}_{i n} \mathcal{F}_{h}, \\
& C_{2}(G)=D_{u, v v}-D_{v, u v}+D_{v v g} \mathcal{F}_{h}^{*} \mathcal{D}_{i m} \mathcal{F}_{h}-D_{u v g} \mathcal{F}_{h}^{*} \mathcal{D}_{i n} \mathcal{F}_{h} .
\end{aligned}
$$

Assuming $f, \mathbf{F} \in C^{k}$ and the surface $\Gamma \in C^{k+2}$, all of the above approximations are $k$-th order accurate. If $f, \mathbf{F}$ and $\Gamma$ are infinitely differentiable, then the convergence rate is superalgebraic.

Proof The formulas are obtained directly from Defintions 1 and 2 , The convergence rate is easily established from the corresponding, well-known properties of the discrete Fourier transform.

Finally, the $L^{2}$ inner product $\left\langle f_{1}, f_{2}\right\rangle_{\Gamma}$ is computed by

$$
\left\langle\vec{f}_{1}, \vec{f}_{2}\right\rangle_{\Gamma, h}=\sum_{k=1}^{N} \sum_{l=1}^{N} f_{1}\left(u_{k}, v_{l}\right) f_{2}\left(u_{k}, v_{l}\right) \sqrt{g\left(u_{k}, v_{l}\right)} .
$$

\section{The Laplace-Beltrami equation}

Let us now consider the problem of solving the Laplace-Beltrami equation

$$
\Delta_{\Gamma} \phi=b
$$

on a toroidal surface $\Gamma$. For this, we first define $\mathcal{M}_{\Gamma}$, the space of mean-zero functions on $\Gamma$ :

$$
\mathcal{M}_{\Gamma}=\left\{f: \Gamma \rightarrow \mathbb{R} \mid\langle f, e\rangle_{\Gamma}=0\right\},
$$

where $e(u, v)$ is the constant function $e(u, v)=1$.

It is well-known that (8) is rank-one deficient, but invertible as a map from $\mathcal{M}_{\Gamma}$ to $\mathcal{M}_{\Gamma}[24,31$. In order to formulate the problem in terms of an invertible linear system, we replace (8) with

$$
\Delta_{\Gamma} \phi+\langle e, \phi\rangle_{\Gamma} e=b,
$$

where, again, $e(u, v)=1$. The left-hand side is easily shown to be an invertible operator on the space of smooth functions. Moreover, (9) has a unique solution if the right-hand side $b \in \mathcal{M}_{\Gamma}$. To see this, note that

$$
\left\langle e, \Delta_{\Gamma} \phi\right\rangle_{\Gamma}+\langle e, \phi\rangle_{\Gamma}\langle e, e\rangle_{\Gamma}=\langle e, b\rangle_{\Gamma}
$$


Since $\Delta_{\Gamma}$ is self-adjoint and annihilates constant functions, we have

$$
0+\langle e, \phi\rangle_{\Gamma}\langle e, e\rangle_{\Gamma}=0
$$

from which $\langle e, \phi\rangle_{\Gamma}=0$.

It remains to develop a finite-dimensional version of (9). For this, we assume $\phi$ and $b$ are discretized as in section 2.2, and let $c(u, v)=\sqrt{g(u, v)}$ with $\vec{c}$ denoting the corresponding discrete vector. It is then easy to see that the discretization of $(9)$ on a uniform mesh takes the form

$$
\left(\Delta_{\Gamma, h}+\vec{c} \vec{c}^{T}\right) \vec{\phi}=\vec{b}
$$

In order to construct a better-conditioned linear system, we will use the inverse of the "flat Laplacian" as a preconditioner and solve

$$
\left(\Delta_{I, h}+\overrightarrow{1}^{\overrightarrow{1}^{T}}\right)^{-1}\left(\Delta_{\Gamma, h}+\vec{c} \vec{c}^{T}\right) \vec{\phi}=\left(\Delta_{I, h}+\overrightarrow{1}^{T}\right)^{-1} \vec{b}
$$

Here, $\Delta_{I, h}$ is the discrete Laplace-Beltrami operator defined above, but for the flat torus, for which the metric tensor $G$ is the identity operator. The vector $\overrightarrow{1}$ denotes the vector of length $N^{2}$, each of whose entries is one.

Remark 1 We note that the operator $\left(\Delta_{\Gamma, h}+\vec{c} \vec{c}^{T}\right)$ can be applied in $O\left(N^{2} \log N\right)$ operations since all the operators in Div $v_{h}$ and $\mathrm{Grad}_{h}$ are either diagonal or involve the discrete Fourier transform, which can be computed by means of the fast Fourier transform (FFT).

The preconditioner $\left(\Delta_{I, h}+\overrightarrow{1} \overrightarrow{1}^{T}\right)^{-1}$ can also be applied in $O\left(N^{2} \log N\right)$ operations since the flat Laplacian $\Delta_{I, h}$ is diagonalized by the Fourier transform and inversion corresponds to multiplication by $-1 /\left(\left(n^{2}+m^{2}\right)\right.$ in the Fourier domain, unless $n=m=0$, in which case we multiply by -1 .

\section{Harmonic vector fields}

As discussed in the introduction, harmonic vector fields are tangential vector fields $\mathbf{F} \in \mathcal{V}_{\Gamma}$ satisfying the system

$$
\begin{array}{r}
\operatorname{Div}(\mathbf{F})=\nabla_{\Gamma} \cdot \mathbf{F}=0 \\
\operatorname{Curl}(\mathbf{F})=\nabla_{\Gamma} \cdot(\mathbf{n} \times \mathbf{F})=0 .
\end{array}
$$

Thus, one method for their computation is to find the nullspace of 112 , which we will denote by $\mathcal{H}(\Gamma)$.

Remark 2 For a surface $\Gamma$ embedded in $\mathbb{R}^{3}$, if $\mathbf{F} \in \mathcal{H}(\Gamma)$, then $\mathbf{n} \times \mathbf{F}$ is in $\mathcal{H}(\Gamma)$ as well. This follows from the fact that $\mathbf{n} \times(\mathbf{n} \times \mathbf{F})=-\mathbf{F}$. Thus, since $\mathcal{H}(\Gamma)$ is two-dimensional, we need only find a single non-trivial element in the nullspace of (12). A second basis vector is then obtained by simply computing the cross-product of the first with $\mathbf{n}$. 
The finite-dimensional version of (12), using the discretization of section 2.2, is

$$
A\left(\begin{array}{l}
\vec{F}^{1} \\
\vec{F}^{2}
\end{array}\right)=\left(\begin{array}{ll}
V_{1}(G) & V_{2}(G) \\
C_{1}(G) & C_{2}(G)
\end{array}\right)\left(\begin{array}{l}
\vec{F}^{1} \\
\vec{F}^{2}
\end{array}\right)=\left(\begin{array}{l}
0 \\
0
\end{array}\right) .
$$

This is a real $2 N^{2} \times 2 N^{2}$ system of equations.

\subsection{Computation of the nullspace}

To find a null vector, we use the method of 36, which we very briefly review here. The method consists of replacing $(12)$, which we denote by $A \overrightarrow{\mathbf{F}}=0$, with

$$
\left(A+R S^{T}\right)\left(\begin{array}{l}
\vec{F}^{1} \\
\vec{F}^{2}
\end{array}\right)=A\left(\begin{array}{l}
\vec{Q}^{1} \\
\vec{Q}^{2}
\end{array}\right)
$$

where $R, S$ are random matrices in $\mathbb{R}^{2 N^{2} \times 2},\left(\begin{array}{c}\vec{Q}^{1} \\ \vec{Q}^{2}\end{array}\right)$ is a random vector in $\mathbb{R}^{2 N^{2}}$, and $S^{T}$ denotes the transpose of $S$.

A probabilistic argument [36] shows that $\left[A+R S^{T}\right]$ is invertible. After the solution of (14), note that $A\left(\begin{array}{l}\vec{Q}^{1} \\ \vec{Q}^{2}\end{array}\right)$ is in the range of $A$, as is $A\left(\begin{array}{c}\vec{F}^{1} \\ \vec{F}^{2}\end{array}\right)$. Since, with probability one, $R$ is not in the range of $A$, it must follow that $S^{T}\left(\begin{array}{l}\vec{F}^{1} \\ \vec{F}^{2}\end{array}\right)=0$. Hence, $\left(\begin{array}{l}\vec{F}^{1}-\vec{Q}^{1} \\ \vec{F}^{2}-\vec{Q}^{2}\end{array}\right)$ is a non-trivial null vector.

In practice, we will solve (14) iteratively, using as a preconditioner the "flat" approximation to $A^{-1}$, namely

$$
\left(\begin{array}{ll}
V_{1}(I) & V_{2}(I) \\
C_{1}(I) & C_{2}(I)
\end{array}\right)^{\dagger}=\left(\begin{array}{cc}
\mathcal{F}_{h}^{*} \mathcal{D}_{i m} \mathcal{F}_{h} & \mathcal{F}_{h}^{*} \mathcal{D}_{i n} \mathcal{F}_{h} \\
-\mathcal{F}_{h}^{*} \mathcal{D}_{i n} \mathcal{F}_{h} & \mathcal{F}_{h}^{*} \mathcal{D}_{i m} \mathcal{F}_{h}
\end{array}\right)^{\dagger}
$$

Here, $V_{1}(I), V_{2}(I), C_{1}(I)$ and $C_{2}(I)$ denote the discrete divergence and curl components from Lemma 1, using the identity operator in place of the metric $G$. We use the pseudoinverse symbol here $(\dagger)$ to indicate that, when $D_{i n}$ or $D_{i m}$ is to be formally inverted, we regularize the matrix by setting any zero diagonal elements to 1 . It is straightforward to verify that the preconditioner in (15) can be applied in $O\left(N^{2} \log N\right)$ operations using the FFT.

To summarize, a basis $\left\{\mathbf{h}_{1}, \mathbf{h}_{2}\right\}$ for the space of harmonic vector fields $\mathcal{H}(\Gamma)$ on a surface $\Gamma$ of genus one can be computed by solving (14) and letting $\mathbf{h}_{1}=\left(\begin{array}{l}\vec{F}^{1}-\vec{Q}^{1} \\ \vec{F}^{2}-\vec{Q}^{2}\end{array}\right)$. We then set $\mathbf{h}_{2}=\mathbf{n} \times \mathbf{h}_{1}$. 
Remark 3 For a surface of revolution parametrized as in (4), the metric tensor takes the simpler form

$$
G=\left(\begin{array}{cc}
r^{\prime}(u)^{2}+z^{\prime}(u)^{2} & 0 \\
0 & r(u)^{2}
\end{array}\right) .
$$

Since the matrix is diagonal and independent of the parameter $v$, it is easy to find a harmonic vector field. Indeed, the divergence and curl operators reduce to

$$
\begin{aligned}
\operatorname{Div}(\mathbf{F}) & =\frac{1}{\sqrt{g}} \partial_{u}\left(\sqrt{g} F^{1}\right)+\partial_{v} F^{2}, \\
\operatorname{Curl}(\mathbf{F}) & =\frac{1}{\sqrt{g}}\left(\partial_{u}\left(G_{v v} F^{2}\right)-G_{u u} \partial_{v} F^{1}\right) .
\end{aligned}
$$

It is straightforward to verify that $\mathbf{h}_{1}=\left(0, \frac{1}{G_{v v}}\right)=\frac{1}{G_{v v}} \partial_{v} \mathbf{x}$ is a harmonic vector field, without the need to solve any system of equations.

\section{The Hodge decomposition}

Suppose now that the method of the previous section has been used to compute a basis $\left\{\mathbf{h}_{1}, \mathbf{h}_{2}\right\}$ for the space of harmonic vector fields $\mathcal{H}(\Gamma)$ on a surface of genus one. Given a tangential vector field $\mathbf{j}$, we seek its orthogonal decomposition

$$
\mathbf{j}=\nabla_{\Gamma} \alpha+\mathbf{n} \times \nabla_{\Gamma} \beta+\mathbf{j}_{H}
$$

where $\mathbf{j}_{H}=c_{1} \mathbf{h}_{1}+c_{2} \mathbf{h}_{2}$, for unknown scalar functions $\alpha, \beta$ and unknown constants $c_{1}, c_{2}$. Taking the surface divergence of either $\mathbf{j}$ or $\mathbf{n} \times \mathbf{j}$, one obtains the two Laplace-Beltrami equations:

$$
\Delta_{\Gamma} \alpha=\nabla_{\Gamma} \cdot \mathbf{j}, \quad \Delta_{\Gamma} \beta=-\nabla_{\Gamma} \cdot(\mathbf{n} \times \mathbf{j}) .
$$

The right hand sides can be computed using the methods of section 2 , and these equations can be solved using the method of section 3. The constants $c_{1}$ and $c_{2}$ are easily computed by solving the $2 \times 2$ linear system

$$
\left(\begin{array}{cc}
\left\langle\mathbf{h}_{1}, \mathbf{h}_{1}\right\rangle_{\Gamma} & \left\langle\mathbf{h}_{1}, \mathbf{h}_{2}\right\rangle_{\Gamma} \\
\left\langle\mathbf{h}_{2}, \mathbf{h}_{1}\right\rangle_{\Gamma} & \left\langle\mathbf{h}_{2}, \mathbf{h}_{2}\right\rangle_{\Gamma}
\end{array}\right)\left(\begin{array}{c}
c_{1} \\
c_{2}
\end{array}\right)=\left(\begin{array}{c}
\left\langle\mathbf{h}_{1}, \mathbf{j}\right\rangle_{\Gamma} \\
\left\langle\mathbf{h}_{2}, \mathbf{j}\right\rangle_{\Gamma}
\end{array}\right)
$$

\section{Numerical Examples}

We illustrate the performance of our methods using a single (non-axisymmetric) surface. For this, Garabedian coordinates [18, 19] provide a parametrization of a stellarator geometry - a surface of genus $\mathfrak{g}=1$ with a central curve, referred to as the magnetic axis, embedded in the interior. This involves three parameters: the poloidal and toroidal angles, 


\begin{tabular}{|c||c|c|c|}
\hline $\mathrm{m} \backslash \mathrm{n}$ & -1 & 0 & 1 \\
\hline \hline-1 & 0.17 & 0.11 & 0 \\
\hline 0 & 0 & 1 & 0.07 \\
\hline 1 & 0 & 4.5 & 0 \\
\hline 2 & 0 & -0.25 & -0.45 \\
\hline
\end{tabular}

Table 1: Parameters $\Delta_{m n}$ describing the surface.

as well as a radius-like parameter $s$. The magnetic axis is a curve which depends only on the toroidal angle $v$ according to the equation

$$
\left\{\begin{array}{l}
x(s, u, v)=r_{0}(v) \cos v \\
y(s, u, v)=r_{0}(v) \sin v \\
z(s, u, v)=z_{0}(v)
\end{array}\right.
$$

where $r_{0}$ and $z_{0}$ are $2 \pi$-periodic functions of $v$. The outer surface of the stellarator is defined by a finite number of trigonometric modes:

$$
\left\{\begin{array}{l}
x(s, u, v)=\sum \Delta_{m, n} \cos ((1-m) u+n v), \\
y(s, u, v)=\sum \Delta_{m, n} \cos ((1-m) u+n v), \\
z(s, u, v)=\sum \Delta_{m, n} \sin ((1-m) u+n v),
\end{array}\right.
$$

where $\left\{\Delta_{m, n}\right\}$ are real coefficients. In between the magnetic axis and the stellarator surface, we have

$$
\left\{\begin{array}{l}
x(s, u, v)=\cos v\left(r_{0}(v)+R(s, u, v)\left(\sum \Delta_{m, n} \cos ((1-m) u+n v)-r_{0}(v)\right)\right), \\
y(s, u, v)=\sin v\left(r_{0}(v)+R(s, u, v)\left(\sum \Delta_{m, n} \cos ((1-m) u+n v)-r_{0}(v)\right)\right), \\
z(s, u, v)=z_{0}(v)+R(s, u, v)\left(\sum \Delta_{m, n} \sin ((1-m) u+n v)-z_{0}(v)\right) .
\end{array}\right.
$$

Note that the radius-like parameter $s$ appears in the scalar "stretching" function $R(s, u, v)$. Thus, if $R(s, u, v)=0$, then the corresponding point lies on the magnetic axis, while if $R(s, u, v)=1$, then the corresponding point lies on the outer surface. For fixed value of $s$ between 0 and 1, the Garabedian representation describes a set of nested surfaces of genus $\mathfrak{g}=1$ around the magnetic axis - each in the form of the desired parametrization (3). We set $r_{0}(v)=4.8+0.1 \cos v, z_{0}(v)=0.1 \sin v, s=0.8, R(s, u, v)=s(1+0.01(1-s) \cos u \sin v)$ and the coefficients $\Delta_{m n}$ as in Table 1. This yields the surface shown in Fig. 1 .

\subsection{The Laplace-Beltrami equation}

In orer to test our Laplace-Beltrami solver, we consider the smooth function

$$
\psi(u, v)=e^{\cos u+\sin v}+e^{\cos (\kappa(u-v))},
$$



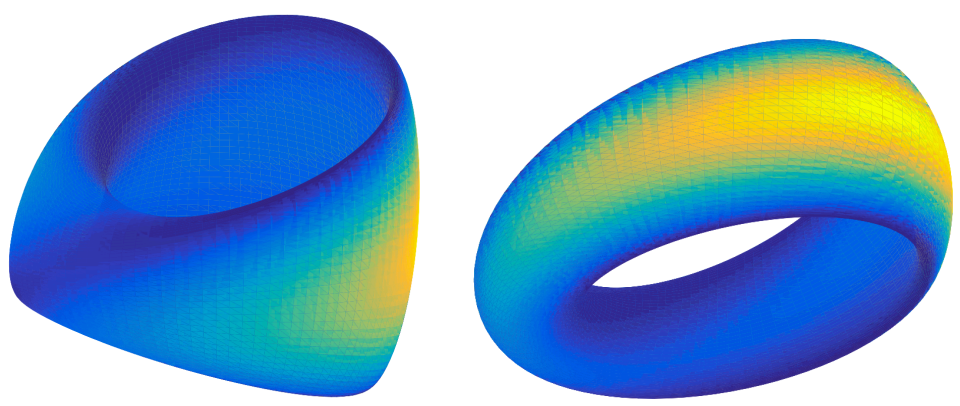

Figure 1: Two views of the genus one surface used in our numerical examples.

\begin{tabular}{|c||c|c|c|}
\hline $\mathrm{N}$ & Iterations & Time & Error \\
\hline 47 & 99 & $0.23 \mathrm{E}+01$ & $0.12 \mathrm{E}+02$ \\
95 & 125 & $0.97 \mathrm{E}+01$ & $0.36 \mathrm{E}-02$ \\
191 & 136 & $0.91 \mathrm{E}+02$ & $0.11 \mathrm{E}-06$ \\
383 & 121 & $0.54 \mathrm{E}+03$ & $0.42 \mathrm{E}-12$ \\
767 & 114 & $0.86 \mathrm{E}+03$ & $0.44 \mathrm{E}-12$ \\
\hline
\end{tabular}

Table 2: Numerical results for the Laplace-Beltrami solver. $N$ denotes the number of points used in the uniform discretization in each component direction (for a total of $N^{2}$ grid points). Also shown are the number of BiCG-stab iterations [37], the solution time and the $L^{2}$ error as a function of $N$.

and the corresponding mean zero function:

$$
\psi_{0}=\psi-\frac{\langle\psi, e\rangle_{\Gamma}}{\langle e, e\rangle_{\Gamma}} e
$$

We then compute the corresponding right hand side as $b(u, v)=\Delta_{\Gamma} \psi_{0}(u, v)$ with $\kappa=12$ and solve

$$
\Delta_{\Gamma, h} \phi=b,
$$

following the method presented in section 3 . Table 2 shows the corresponding convergence results. The error is defined as $\left\|\phi-\psi_{0}\right\|_{2}$.

\subsection{Harmonic vector fields}

We next compute the harmonic vector fields on the surface, following the method described in section 4 . Table 3 displays the corresponding convergence results. 


\begin{tabular}{|c||c|c|c|c|}
\hline $\mathrm{N}$ & Iterations & Time & Div error & Curl error \\
\hline 11 & 85 & $0.13 \mathrm{E}+00$ & $0.24 \mathrm{E}-02$ & $0.27 \mathrm{E}-02$ \\
23 & 115 & $0.86 \mathrm{E}+00$ & $0.18 \mathrm{E}-04$ & $0.18 \mathrm{E}-04$ \\
47 & 112 & $0.40 \mathrm{E}+01$ & $0.92 \mathrm{E}-08$ & $0.93 \mathrm{E}-08$ \\
95 & 129 & $0.15 \mathrm{E}+02$ & $0.14 \mathrm{E}-11$ & $0.31 \mathrm{E}-11$ \\
191 & 154 & $0.17 \mathrm{E}+03$ & $0.13 \mathrm{E}-11$ & $0.15 \mathrm{E}-11$ \\
383 & 163 & $0.12 \mathrm{E}+04$ & $0.43 \mathrm{E}-11$ & $0.45 \mathrm{E}-11$ \\
767 & 189 & $0.20 \mathrm{E}+04$ & $0.47 \mathrm{E}-10$ & $0.48 \mathrm{E}-10$ \\
\hline
\end{tabular}

Table 3: Numerical results for the computation of harmonic vector fields. $N$ denotes the number of points used in the uniform discretization in each component direction (for a total of $N^{2}$ grid points). Also shown are the number of BiCG-stab iterations [37, the solution time and the $L^{2}$ error in the surface divergence and surface curl as a function of $N$.

\begin{tabular}{|c||c|c|c|}
\hline $\mathrm{N}$ & Iterations & Time & Error \\
\hline 47 & 112 & $0.12 \mathrm{E}+02$ & $0.21 \mathrm{E}+00$ \\
95 & 129 & $0.62 \mathrm{E}+02$ & $0.26 \mathrm{E}-05$ \\
191 & 154 & $0.51 \mathrm{E}+03$ & $0.25 \mathrm{E}-11$ \\
383 & 163 & $0.36 \mathrm{E}+04$ & $0.42 \mathrm{E}-13$ \\
767 & 189 & $0.10 \mathrm{E}+05$ & $0.38 \mathrm{E}-13$ \\
\hline
\end{tabular}

Table 4: Numerical results for the Hodge decomposition. $N$ denotes the number of points used in the uniform discretization in each component direction (for a total of $N^{2}$ grid points). Also shown are the number of BiCG-stab iterations [37], the time to find a basis for the harmonic vector fields and solve the two Laplace-Beltrami equations, and the $L^{2}$ error in the reconstructed vector field as a function of $N$.

\subsection{The Hodge decomposition}

Finally, we consider a vector field in the ambient three-dimensional space given by $\mathbf{j}(\mathbf{x})=$ $\nabla(\sin \imath \kappa \mathbf{k} \cdot \mathbf{x})+\mathbf{n} \times \nabla(\sin \imath \kappa \mathbf{k} \cdot \mathbf{x})$ with $\kappa=10$ and $\mathbf{k}=(0,0,1)$. We define its tangential component $\mathbf{j}_{T}$ on the stellarator surface, and compute its Hodge decomposition using the method presented in section 5. This requires solving two Laplace-Beltrami equations to compute the curl-free component $\left(\nabla_{\Gamma} \alpha\right)$ and the divergence free component $\left(\mathbf{n} \times \nabla_{\Gamma} \beta\right)$. It also requires a basis for the harmonic vector fields to determine the harmonic component $\mathbf{j}_{H}$. Table 4 presents the corresponding convergence results. The error is computed using the $L_{2}$ norm induced by the inner product for tangential vector fields in (6), which we denote by $\left\|\mathbf{j}_{T}-\nabla_{\Gamma} \alpha-\mathbf{n} \times \nabla_{\Gamma} \beta-\mathbf{j}_{H}\right\|_{2}$. The vector field and its various components are shown in Figure 2 . 

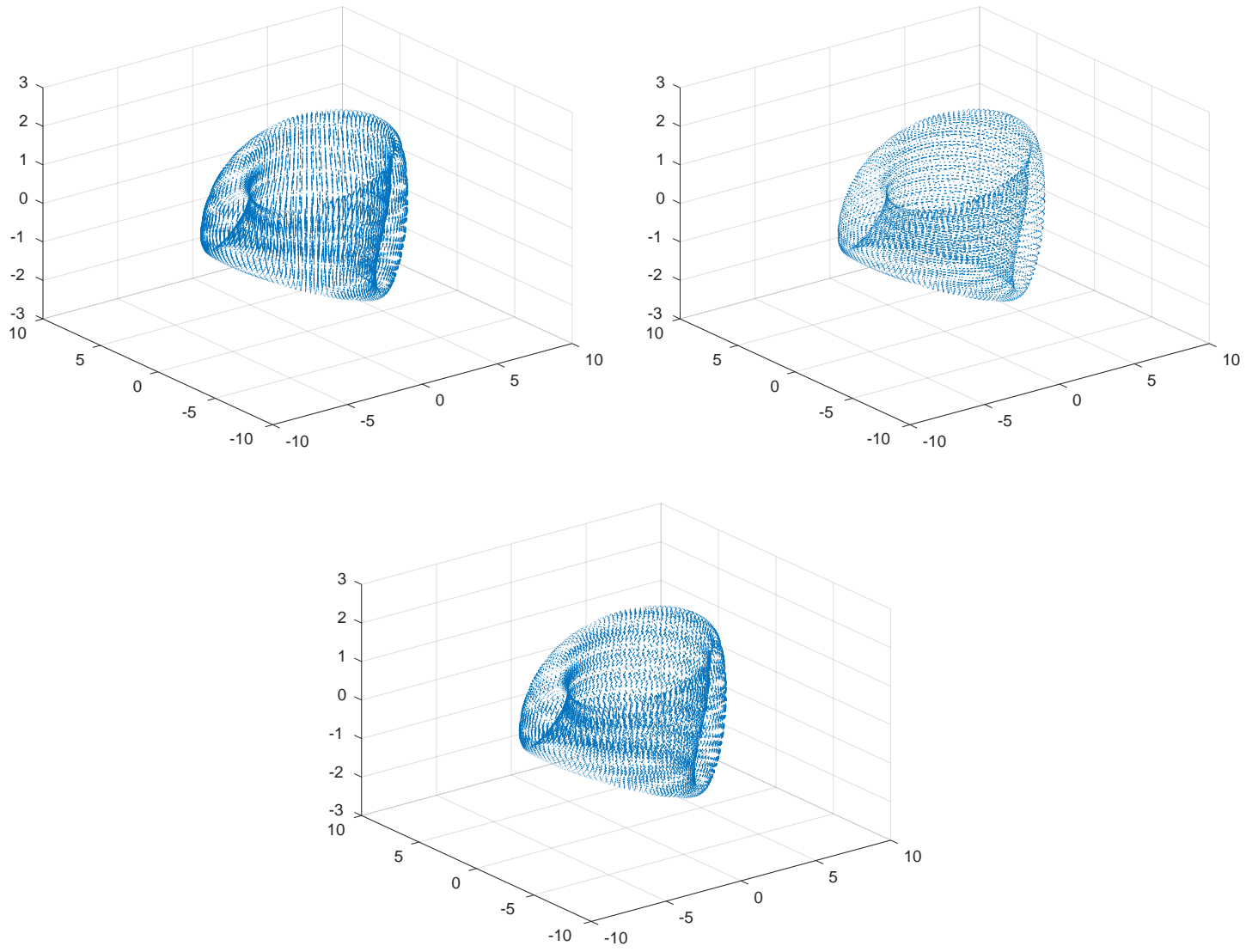

Figure 2: The various components of the Hodge decomposition of the vector field $\mathbf{j}(\mathbf{x})=$ $\nabla(\sin \imath \kappa \mathbf{k} \cdot \mathbf{x})+\mathbf{n} \times \nabla(\sin \imath \kappa \mathbf{k} \cdot \mathbf{x})$ : its curl-free component $\mathbf{n} \times \nabla_{\Gamma} \beta$ (top left), its divergence free component $\nabla_{\Gamma} \alpha$ (top right), and its harmonic component $\mathbf{j}_{H}$ (bottom). The vector fields are rescaled for clarity. 


\section{Conclusions}

We have presented a simple but effective pseudo-spectral method for solving the LaplaceBeltrami equation on surfaces of genus one, and for determining a basis for surface harmonic vector fields. This permits the fast and accurate computation of the full Hodge decomposition of a given smooth tangential vector field. Our method relies on the FFT and a "flat torus" preconditioner, requiring $O\left(N^{2} \log N\right)$ work where $N^{2}$ is the number of points in the surface representation.

There are several drawbacks to our scheme: it relies on a global parametrization, it is inherently non-adaptive, and it is limited in its present form to surfaces of genus one. We are currently exploring variants of our approach that will be able to handle these generalizations.

\section{References}

[1] D. Adalsteinsson and J. Sethian. A level set approach to a unified model for etching, deposition, and lithography. Journal of Computational Physics, 138:193-223, 1997.

[2] D. N. Arnold, R. S. Falk, and R. Winther. Finite element exterior calculus: from Hodge theory to numerical stability. Bulletin of the American Mathematical Society, 47:281-354, 2010.

[3] C. V. Atanasiu, A. H. Boozer, L. E. Zakharov, A. A. Subbotin, and G. I. Miron. Determination of the vacuum field resulting from the perturbation of a toroidally symmetric plasma. Physics of Plasmas, 6:2781-2790, 1999.

[4] M. Belkin, J. Sun, and Y. Wang. Discrete Laplace operator on meshed surfaces. In Proceedings of the Twenty-fourth Annual Symposium on Computational Geometry, SCG '08, pages 278-287, New York, NY, USA, 2008. ACM.

[5] M. Bertalmio, L. Cheng, S. Osher, and G. Sapiro. Variational problems and partial differential equations on implicit surfaces. Journal of Computational Physics, 174:759 $780,2001$.

[6] S.-G. Chen, M.-H. Chi, and J.-Y. Wu. High-order algorithms for Laplace-Beltrami operators and geometric invariants over curved surfaces. Journal of Scientific Computing, 65:839-865, 2015.

[7] E. V. Chernokozhin and A. Boag. Method of generalized Debye sources for the analysis of electromagnetic scattering by perfectly conducting bodies with piecewise smooth boundaries. IEEE Transactions on Antennas and Propagation, 61:2108-2115, 2013. 
[8] R. R. Coifman, S. Lafon, A. B. Lee, M. Maggioni, B. Nadler, F. Warner, and S. W. Zucker. Geometric diffusions as a tool for harmonic analysis and structure definition of data: Diffusion maps. Proceedings of the National Academy of Sciences of the United States of America, 102:7426-7431, 2005.

[9] D. Colton and R. Kress. Inverse Acoustic and Electromagnetic Scattering Theory, Applied Mathematical Sciences 93. Springer, New York, 1998.

[10] W. D'haeseleer, W. Hitchon, J. Callen, and J. Shohet. Flux coordinates and magnetic field structure. Springer Verlag, Berlin, 1991.

[11] L. R. Duduchava, D. Mitrea, and M. Mitrea. Differential operators and boundary value problems on hypersurfaces. Mathematische Nachrichten, 279:996-1023, 2006.

[12] G. Dziuk and C. M. Elliott. Finite element methods for surface PDEs. Acta Numerica, $22: 289-396,2013$.

[13] C. L. Epstein and L. Greengard. Debye sources and the numerical solution of the time harmonic Maxwell equations. Communications on Pure and Applied Mathematics, 63:413-463, 2010.

[14] C. L. Epstein, L. Greengard, and M. O'Neil. Debye sources and the numerical solution of the time harmonic Maxwell equations II. Communications on Pure and Applied Mathematics, 66:753-789, 2013.

[15] C. L. Epstein, L. Greengard, and M. O’Neil. Debye sources, Beltrami fields and a complex structure on Maxwell fields. Communications on Pure and Applied Mathematics, 68:2237-2280, 2015.

[16] M. Fisher, P. Schröder, M. Desbrun, and H. Hoppe. Design of tangent vector fields. ACM Trans. Graph., 26:56:1-56:9, 2007.

[17] P. R. Garabedian. Partial differential equations. John Wiley \& Sons, Inc., New YorkLondon-Sydney, 1964.

[18] P. R. Garabedian. A method of canonical coordinates for flow computations. Communications on Pure and Applied Mathematics, 23(3):313-327, 1970.

[19] P. R. Garabedian. Three-dimensional analysis of tokamaks and stellarators. Proceedings of the National Academy of Sciences, 105:13716-13719, 2008.

[20] P. W. Gross and P. R. Kotiuga. Electromagnetic Theory and Computation: A Topological Approach. MSRI Publications - Volume 48, Cambridge University Press, New York, NY, 2004. 
[21] X. Gu and S.-T. Yau. Computing conformal structures of surfaces. Communications in Information and Systems, 2:121-146, 2002.

[22] K. Hildebrandt and K. Polthier. On approximation of the Laplace-Beltrami operator and the Willmore energy of surfaces. Computer Graphics Forum, 30:1513-1520, 2011.

[23] G. Huiskamp. Difference formulas for the surface Laplacian on a triangulated surface. Journal of Computational Physics, 95:477-496, 1991.

[24] J. Jost. Riemannian Geometry and Geometric Analysis. Springer Verlag, Berlin, 2011.

[25] M. C. A. Kropinski and N. Nigam. Magnetic energy, multiply connected domains, and force-free fields. Phys. Rev. A, 46:2177-2123, 1992.

[26] M. C. A. Kropinski and N. Nigam. Fast integral equation methods for the LaplaceBeltrami equation on the sphere. Advances in Computational Mathematics, 40:577$596,2014$.

[27] C. B. Macdonald, J. Brandman, and S. J. Ruuth. Solving eigenvalue problems on curved surfaces using the closest point method. J. Comput. Phys., 230:7944-7956, 2011 .

[28] J.-C. Nédélec. Acoustic and Electromagnetic Equations. Springer, New York, 2001.

[29] M. A. Olshanskii, A. Reusken, and J. Grande. A finite element method for elliptic equations on surfaces. SIAM Journal on Numerical Analysis, 47:3339-3358, 2009.

[30] F. Petronetto, A. Paiva, M. Lage, G. Tavares, H. Lopes, and T. Lewiner. Meshless Helmholtz-Hodge decomposition. IEEE Transactions on Visualization and Computer Graphics, 16:338-349, March 2010.

[31] S. Rosenberg. The Laplacian on a Riemannian Manifold. Cambridge University Press, New York, NY, 1997.

[32] S. J. Ruuth and B. Merriman. A simple embedding method for solving partial differential equations on surfaces. Journal of Computational Physics, 227:1943-1961, 2008 .

[33] G. Sapiro. Geometric Partial Differential Equations and Image Analysis. Cambridge University Press, New York, NY, 2001.

[34] P. Schwartz, D. Adalsteinsson, P. Colella, A. P. Arkin, and M. Onsum. Numerical computation of diffusion on a surface. Proceedings of the National Academy of Sciences of the United States of America, 102:11151-11156, 2005. 
[35] G. Schwarz. Hodge Decomposition - A Method for Solving Boundary Value Problems. Springer Verlag, New York, 1995.

[36] J. Sifuentes, Z. Gimbutas, and L. Greengard. Randomized methods for rank-deficient linear systems. Electronic Transactions on Numerical Analysis, 44:177-188, 2015.

[37] H. A. van der Vorst. Bi-CGSTAB: A fast and smoothly converging variant of bi-CG for the solution of nonsymmetric linear systems. SIAM Journal on Scientific and Statistical Computing, 13:631-644, 1992.

[38] F. W. Warner. Foundations of differentiable manifolds and Lie groups. Springer, New York, 1983.

[39] K. Xu, H. Zhang, D. Cohen-Or, and Y. Xiong. Dynamic harmonic fields for surface processing. Computers \& Graphics, 33:391-398, 2009. 